\title{
AN INVERSE METHOD FOR DETERMINING PARAMETERS FOR FOLDED STRUCTURES
}

\author{
BY
}

\author{
I. LERCHE
}

\begin{abstract}
Department of Geological Sciences, University of South Carolina, Columbia, South Carolina
\end{abstract}
\begin{abstract}
General analytic solutions are presented here to an equation relating two angles describing fault shape, and two angles describing fold shape. The analytic solutions provide any of the four angles when the other three are given.

Armed with the general analytic formulae (including generalizations of the original equation to relate present-day sedimentary bed positions to fault properties which have also been previously given), an inverse procedure is then developed which allows parameter estimates for fault and bed properties to be determined, while honoring the present day data.

The inverse method is guaranteed to keep all parameters within pre-set chosen bounds, and guarantees to find a minimum mismatch between predicted and observed present day bed locations and fault position. In this way an appreciation can be achieved of the assumptions, parameters, and data quality, quantity and distribution, in relation to resolution, precision, uniqueness, and sensitivity of model outputs and parameters being sought.
\end{abstract}

I. Introduction. One of the more obvious facts about geological problems in general is that only present-day information is available. Thus, any determination of the evolution with time and space of a particular geological problem has to rely upon inferences from present-day information.

In turn, such inferences normally require that some model be constructed of proposed behavior, with the attendant concerns of assumptions intrinsic to the model, of assumption (or specification of) parameter values in the model, and of the quality, quantity, and sampling frequency of data used as control information for the model. The outcome from any model must then be evaluated for its uniqueness, resolution, sensitivity, and precision in respect of honoring the available information.

Two possible avenues are available in such evolutionary model scenarios: one can construct a "forward" model in which initial and/or boundary conditions are specified within the broad framework of the intrinsic assumptions of the model; then the evolution of the model can be carried through, finally arriving at a present-day model result.

Received February 24, 1994.

1991 Mathematics Subject Classification. Primary 86A22, 86A60. 
Alternatively, one can start with the present-day information and use inverse procedures to determine (or at least to constrain) the paleo-evolution of the model in respect to its underlying assumptions, functional dependences, and parameter values.

A third approach, and the one usually taken in geological problems, is to perform a "pseudo-inverse", in which the intrinsic assumptions of the model are adhered to, as are the generic functional dependences, but the parameter values (which control the basic response patterns) are constrained or determined by arranging for the model to honor to the best of its ability, both the present-day data as well as any paleo-constraints (e.g., horizontal sedimentary beds at deposition) imposed on the system.

This third procedure, and applied mathematical methods which guarantee that parameter values can indeed be determined, is the focus of this paper.

The vehicle used to illustrate the general method of application is the problem of roll-over faults.

Often, a sedimentary section is observed to be faulted, with a down-thrown sedimentary section on one side of the fault. The down-thrown section may indicate an instantaneous fault event (observed throws identical for all sedimentary beds of different ages) or may indicate a continuously active growth fault (observed throws of older sedimentary beds progressively larger with greater depth of burial).

The volume accommodation problem (lateral motion of the down-thrown sedimentary beds) often leads to beds that "roll-over" against the fault, which itself is curved.

Procedures for reconstructing the motion of the sedimentary beds in respect to their observed positions along the fault then rely upon specifying (a) the geometry of the fault and whether that geometry remains invariant for all time; (b) the requirements for the faulted sedimentary beds at the times of their deposition on both sides of the fault. Down-thrown sediments are customarily referred to as being on the "hanging wall" side of the fault, while the remaining sediments are said to be on the "footwall" side; (c) the volume, or area, requirements of the sediments with time (e.g., are the sedimentary formations treated as of fixed volume or are geometrical thicknesses compacted with time from their depositional values to allow for the changes due to water loss from the sediments?).

Accordingly, the reconstruction of evolving sedimentary structural patterns of behavior is by no means a simple problem, being controlled by the model assumptions and model parameters.

Here we investigate the mathematical methods that can be used to infer parameter values for any model. The vehicle we choose as an illustrative case is the simple situation occurring when (a) the fault curve shape is the same for all time; (b) the footwall sediments move only vertically; (c) compaction is ignored.

For this model situation Xiao and Suppe $(1989,1992)$ have recently presented an analysis of the development of roll-over structures against a rigid footwall, in which the fault surface is composed of two planes meeting at a kink point. Reproduced here as Figures 1 and 2 are representations of the geometrical scheme considered, with angle nomenclature and definitions of sediment behaviors taken directly from Xiao and Suppe (1989, 1992) and Lorenzetti and Brennan (1993), so that a common frame of reference is available for discussion. 
It is clear that for specified values of any three of roll-over dip angle $\delta$, fault cut-off angle $\theta$, fault bed angle $\phi$, or fault cut-off angle on the lower fault segment $(\theta-\phi)$, together with specification of the sedimentation to subsidence ratio, SSR, and one scale length, $L$ (e.g., the distance along the fault to the kink bend), then the locations and structural deformations of sedimentary beds impacted by the fault can be drawn. This sort of pattern of roll-over fault and sediment development has been considered by Xiao and Suppe (1992), and explored extensively in graphical form by Lorenzetti and Brennan (1993). A good appreciation of the patterns of development, and of the relations of different angle factors to each other is presented in the large number of numerical cases presented in Lorenzetti and Brennan (1993). There is no need to repeat this exhaustive exercise. The forward problem (i.e., with parameters specified), under the conditions and assumptions laid down by Xiao and Suppe (1992), would then seem to be well understood after the work of Lorenzetti and Brennan (1993).

Accordingly, with prior specification of the fault bend position, fault length of each section, sedimentation to subsidence ratio, and angles, the behavior and motion of sedimentary beds (both pre-fault, syn-fault, and post-fault) can be determined by pure geometric considerations and with prescribed sediment deposition rates through time. The result is a two-dimensional section at the present day which owes its geometric form to (a) the assumptions of the model (e.g., fixed footwall, no compaction); (b) the particular values for angles specified in the system. Presumably of concern to an understanding of the likely evolution of an observed set of present-day data are (i) the ability to investigate how well this particular model of folded structures matches to observed behaviors; (ii) the ability to determine the particular values of parameters (and their uncertainties) which most closely allow a match between model predictions and observed behavior; and (iii) the ability to determine the degrees of resolution, uniqueness, sensitivity, and uncertainty of paleo-evolution from present-day information.

The aim in this paper is to provide precisely those procedures which not only honor present-day observations, but which also allow the use of observations to determine the parameter values (and their uncertainties) which provide minimum discord of observations and predictions.

Three mathematical components need to be discussed: (i) to provide analytic solutions to Eq. (4) of Xiao and Suppe (1992), who remarked that the equation was not solvable analytically; (ii) to use generalizations of the original Xiao and Suppe (1992) fault angle equation obtained by Lorenzetti and Brennan (1993) to relate present-day sedimentary bed positions to fault properties; (iii) to provide an inverse procedure so that the difference between predicated and observed present-day bed positions can be used to obtain parameter estimates that most closely allow satisfaction of the observed bed and fault positions.

II. Solutions to Equation (4) of Xiao and Suppe (1992). With reference to Fig. 1, the relation between the two angles $\theta$ and $\phi$ describing the fault shape, and the two angles $\delta$ and $\psi$ describing the fold shape is given by Xiao and Suppe (1992) (their Eq. 4) as

$$
\sin \psi \sin (\psi-\delta) \operatorname{cosec}(\theta+\psi-\phi) \operatorname{cosec}(\theta+\psi)=\sin \delta / \sin \phi
$$




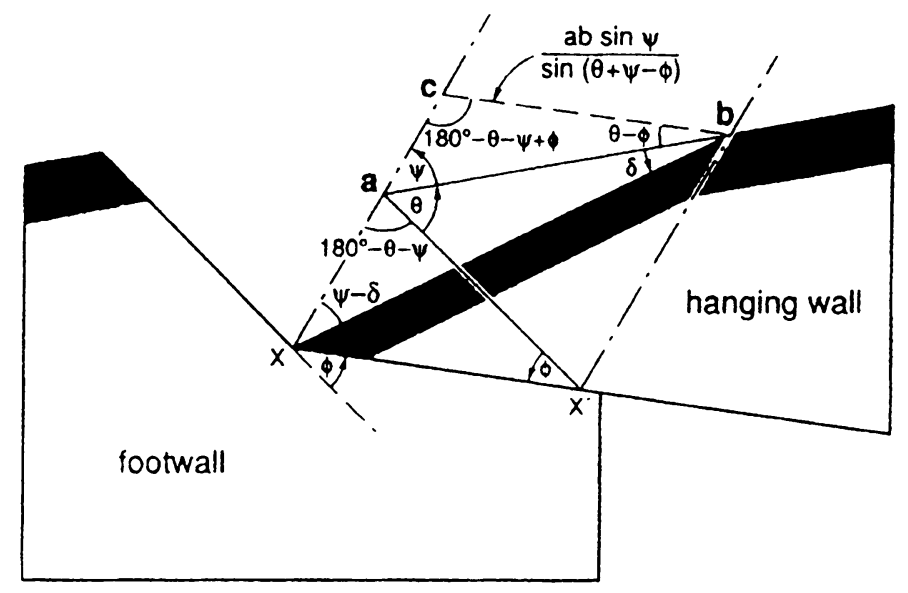

Figure 1. A direct reproduction of Fig. 4 of Xiao and Suppe (1992) who note that the figure represents "Geometric relationship between fault shape and rollover shape for a single sharp fault bend. Hangingwall collapse is in the axial surface direction $\left(a x\right.$ and $\left.b x^{\prime}\right)$. The undeformed shape of the dipping beds in $\Delta b x x^{\prime}$ is $\Delta a b x^{\prime} . "$

Xiao and Suppe (1992) remark, "This is an implicit equation with no analytic solutions; it must be solved numerically." The purpose of this short section is to show that given any three of the four angles $(\psi, \delta, \theta, \phi)$, Eq. (1) always admits of an analytic solution for the fourth angle.

Four cases are available for Eq. (4):

(i) given $\psi, \phi, \delta$, determine $\theta$,

(ii) given $\theta, \psi, \delta$, determine $\phi$,

(iii) given $\theta, \psi, \phi$, determine $\delta$,

(iv) given $\theta, \phi, \delta$, determine $\psi$.

Consider each in turn.

(i) Determine $\theta$, given $\psi, \phi$, and $\delta$. Rewrite Eq. (1) as

$$
\sin (\theta+\psi-\phi) \sin (\theta+\psi)=\sin \psi \sin (\psi-\delta) \sin \phi / \sin \delta
$$

The left-hand side of Eq. (2) can be written $\frac{1}{2}[\cos \phi-\cos (2(\theta+\psi)-\phi)]$; so it follows that

$$
\theta=-\psi+\frac{1}{2} \phi+\frac{1}{2} \cos ^{-1}\{\cos \phi-2 \sin \psi \sin (\psi-\delta) \sin \phi / \sin \delta\}
$$

which expresses $\theta$ analytically in terms of $\psi, \phi$, and $\delta$.

(ii) Determine $\phi$, given $\phi, \psi$, and $\delta$. Rewrite Eq. (1) as

$$
\sin (\theta+\psi-\phi) / \sin \phi=\sin \psi \sin (\psi-\delta) /(\sin \delta \sin (\theta+\psi))
$$

The left-hand side of Eq. (4) can be written $\sin (\theta+\psi) \cot \phi-\cos (\theta+\psi)$; so it follows that

$$
\phi=\tan ^{-1}\left\{\frac{\sin ^{2}(\theta+\psi) \sin \delta}{[\sin \psi \sin (\psi-\delta)+\sin \delta \sin (\theta+\psi) \cos (\theta+\psi)]}\right\},
$$

which expresses $\phi$ analytically in terms of $\theta, \psi$, and $\delta$. 
(iii) Determine $\delta$, given $\theta, \psi$, and $\phi$. Rewrite Eq. (2) as

$$
\sin (\psi-\delta) / \sin \delta=\sin (\theta+\psi-\phi) \sin (\theta+\psi) /(\sin \psi \sin \phi)
$$

The left-hand side of Eq. (6) can be written as $\sin \psi \cot \delta-\cos \psi$; so it follows that

$$
\delta=\tan ^{-1}\left\{\frac{\sin ^{2} \psi \sin \phi}{[\sin (\theta+\psi-\phi) \sin (\theta+\psi)+\cos \psi \sin \psi \sin \phi]}\right\}
$$

which expresses $\delta$ analytically in terms of $\theta, \psi$, and $\phi$.

(iv) Determine $\psi$, given $\theta, \phi$, and $\delta$. Rewrite Eq. (1) as

$$
[\sin (\psi-\delta) \sin \psi] \sin \phi=[\sin (\theta+\psi-\phi) \sin (\theta+\psi)] \sin \delta
$$

which can be written

$$
[\cos \delta-\cos (2 \psi-\delta)] \sin \phi=[\cos \phi-\cos (2 \psi+2 \theta-\phi)] \sin \delta
$$

Then rewrite Eq. (9) as

$$
\begin{aligned}
& \cos 2 \psi[-\cos \delta \sin \phi+\sin \delta \cos (2 \theta-\phi)] \\
& \quad-\sin 2 \psi[+\sin \phi \sin \delta+\sin \delta \sin (2 \theta-\phi)]+\sin (\phi-\delta)=0 .
\end{aligned}
$$

It then follows that

$$
\psi=-\frac{1}{2} r+\frac{1}{2} \cos ^{-1}[\sin (\delta-\phi) / u]
$$

where

$$
u=\left(\sin ^{2} \delta+\sin ^{2} \phi-2 \sin \phi \sin \delta \cos (2 \theta+\delta-\phi)\right)^{1 / 2}
$$

and

$$
r=\tan ^{-1}\left\{\frac{[\sin \phi \sin \delta+\sin \delta \sin (2 \theta-\phi)]}{[\sin \delta \cos (2 \theta-\phi)-\cos \delta \sin \phi]}\right\}
$$

so that $\psi$ is expressed analytically in terms of $\theta, \phi$, and $\delta$.

Thus, in all four cases exact analytic expressions are, in fact, available.

Within the framework of the model for folded structures proposed by Xiao and Suppe (1992), it follows that once three of the angles are specified, the fourth is uniquely determined.

As we shall show in Sec. V, which deals with an inverse procedure, it is possible to both use and also to ignore the angle requirements of the model in order to see how well the model is constrained by the data. 
III. The Lorenzetti-Brennan (1993) generalization. Lorenzetti and Brennan (1993) have related the growth axial angle $\gamma$ (see Fig. 2) to the angles $\theta, \phi$, and $\psi$ and also to the sedimentation to subsidence ratio, SSR, defined as the ratio of the thickness of hanging-wall sediments near the fault to the vertical component of slip on the upper fault segment (see Fig. 2). Then angle $\gamma$ is given through Eq. (9) of Lorenzetti and Brennan (1993) as

$$
\cot \gamma=[\sin (\theta+\psi) \sin (\theta-\phi+\psi) / A]-\cot \psi
$$

where

$$
A=\sin \psi\{(\mathrm{SSR}-1) \sin \theta \sin (\theta-\phi+\psi)+\sin (\theta+\psi) \sin (\theta-\phi)\} .
$$

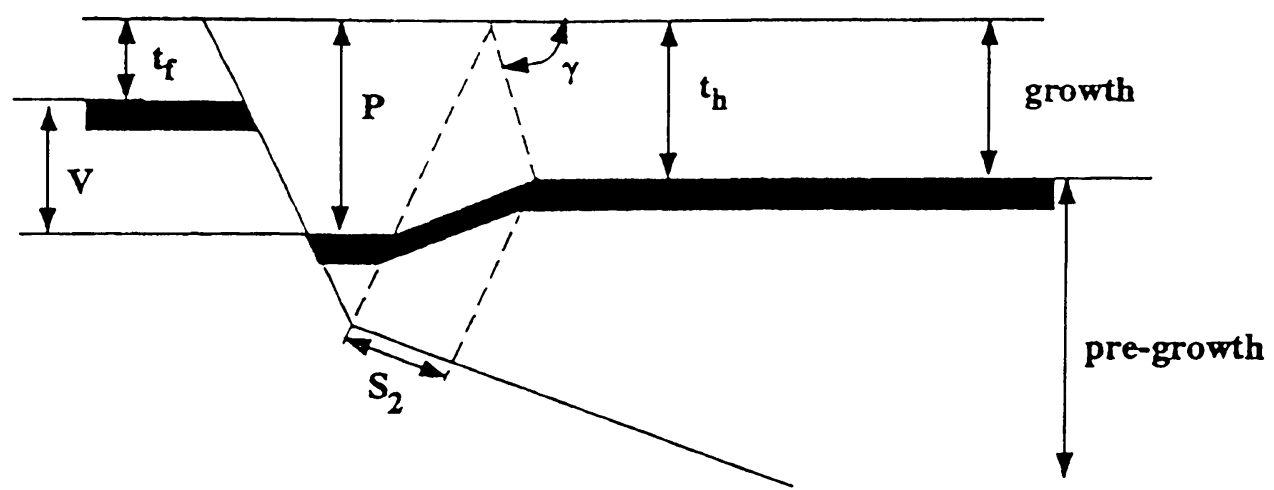

Expansion index $(E)=t_{h} / t_{f}$

Dimensionless growth rate $(D G R)=S_{2} / t_{h}$

Sediment to subsidence ratio $(\mathrm{SSR})=\mathrm{P} / \mathrm{V}$

Expansion ratio $(E R)=P / t_{f}$

Growth ratio $(G R)=P / t_{h}$

$t_{f}=$ footwall thickness of growth sediments

$\mathrm{P}=$ hanging wall thickness of growth sediments near fault

$t_{h}=$ "regional" hanging wall growth sediment thickness

$S_{2}=$ slip below the fault bend

$\mathrm{V}=$ vertical component of slip on upper fault segment

(a)

Figure 2a. A direct reproduction of Fig. 18(a) of Lorenzetti and Brennan (1993) who note, "Some commonly used measures of relative stratal thicknesses across normal faults. In the equations derived by Xiao and Suppe (1992), expansion index (EI) is used. This quantity is defined as the ratio of the undeformed growth sediment thickness in the hanging wall to the growth sediment thickness in the footwall block. Other measures, shown here, are related to the growth axial angle $(\gamma)$." 


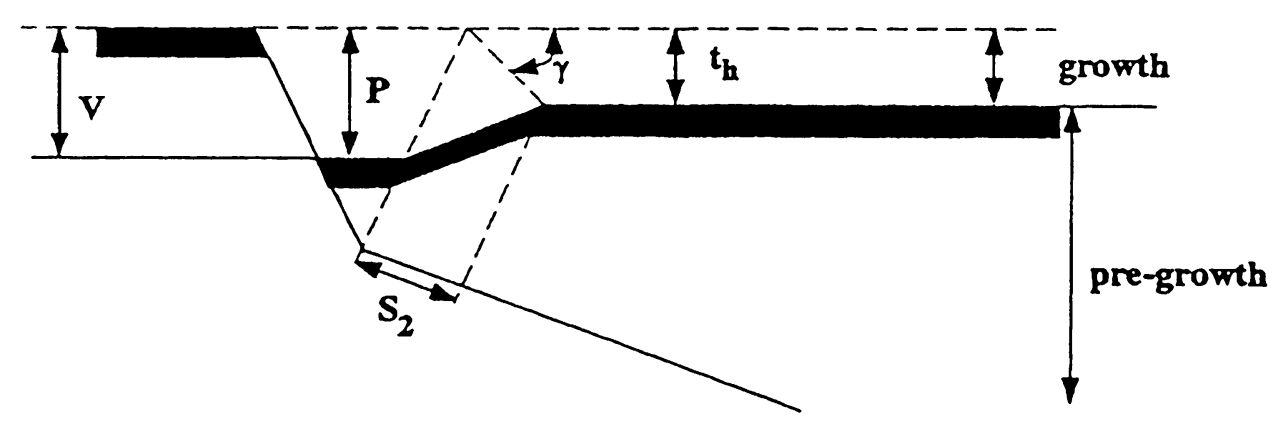

(b)

Figure 2b. A direct reproduction of Fig. 18(b) of Lorenzetti and Brennan (1993) who note, "At low relative sedimentation rates, $t_{f}$ goes to zero, the growth axial angle $(\gamma)$ becomes smaller, and the expansion index (EI) and expansion ratio (ER) are undefined. In this diagram, SSR $=1.0$ (the basin created by the normal fault is filled with sediment exactly as fast as the basin is being formed, and there is no growth sediment on the footwall; $P=V)$."

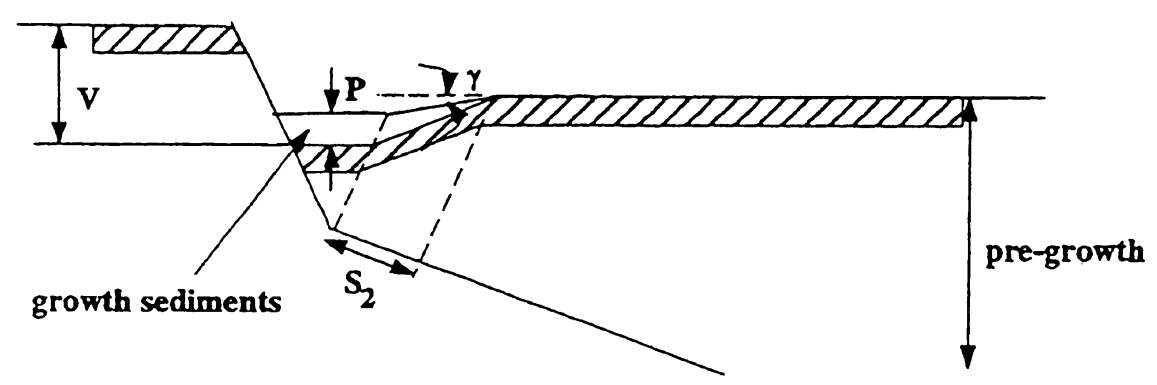

(c)

Figure 2c. A direct reproduction of Fig. 18(c) of Lorenzetti and Brennan (1993) who note, "At the very lowest sedimentation to subsidence ratios, $t_{h}$ goes to zero, $\gamma$ changes sign, the growth rate (GR) and the dimensionless growth ratio (DGR) are undefined. Only the SSR is still valid, and the restricted nature of the basin is indicated by the fact that $P<V$, and hence the SSR is less than 1.0."

IV. Inverse Procedures. The problem to be addressed here is the inverse of the forward problem. Basically the argument is as follows: on seismic sections (migrated to true depth sections) one observes the present-day locations of sedimentary beds and of a fault bend. The question then is: can one use the observed bed locations to infer the parameter values of the model most consistent with the observations, and can one obtain measures of precision, resolution, uniqueness, and uncertainty on the available parameters, so the limitations can be set on the possible ranges of extensional structural developments with time?

The purpose of this section of the paper is to demonstrate that such a goal is achievable and to provide quantitative procedures for determining, or at least bracketing, the 
relevant parameters controlling the system under the model assumptions as set down by Xiao and Suppe (1992).

Imagine first that a forward model were to be run. Controlling parameters are (i) three angles (say $\phi, \psi$, and $\delta$ to fix ideas) with the fourth determined by the analytic solutions to Xiao and Suppe's equation (4); (ii) the sedimentation rate to subsidence rate ratio, SSR, from which the growth axial angle is then determined using Eq. (9) of Lorenzetti and Brennan (1993), which involves SSR and the angles of Eq. (4) of Xiao and Suppe (1992); (iii) a scale length, $L$, which measures either depth, length to a fault kink, etc. In short, five parameters, $\phi, \psi, \delta$, SSR, and $L$ describe the evolution of the system relative to a given depositional behavior for the sedimentary beds (horizontal in the classical model of Xiao and Suppe (1992); non-horizontal, but of fixed slope, in the generalization of Lorenzetti and Brennan (1993)).

After evolution from fault initiation time $t=t_{f}$ until the present day, $t=0$, a sequence of $N$ sedimentary beds $(B=1, \ldots, N)$ will then occupy a set of locations with the present-day location of the $B$ th bed described through $Y=Y_{B}(x, p)$, where the vertical coordinate is $Y$ and the lateral coordinate is $x$. The vector $p$ is the vector of all five parameters $(\psi, \phi, \delta, \mathrm{SSR}, L)$ describing the system.

Thus, for a given initial choice of the components of the vector $p$, all sedimentary bed positions will be determined at present day. However, direct measurements are available for the observed beds at $Y=Y_{B}\left(x_{i}\right)$ at $R$ lateral positions $x_{i}(i=1, \ldots, R)$, where the vertical coordinate reference is the same as for the forward run model calculation.

With arbitrary choices of the vector $p$ there is no reason why model predictions $Y_{B}\left(x_{i}, p\right)$ and observations $Y_{B}\left(x_{i}\right)$ at each location $x_{i}$ and for each bed $B$ should be even close to each other. The aim is to use the difference between observed and predicted behaviors to provide a procedure which iteratively corrects the model parameters towards values leading to the least discord between predictions and observations. Such a procedure operates as follows. The five parameters in the vector $p$ have different dimensions. Let the $j$ th component of $p$ have a maximum value $\max _{j}$ and a $\operatorname{minimum}$ value $\min _{j}$. Then set

$$
a_{j}=\left(p_{j}-\min _{j}\right) /\left(\max _{j}-\min _{j}\right)
$$

so that $0 \leq a_{j} \leq 1$ and $\min _{j} \leq p_{j} \leq \max _{j}$. Regard the vector $a$ as fundamental with $p$ being a dependent vector given through

$$
p_{j}=\min _{j}+a_{j}\left(\max _{j}-\min _{j}\right) .
$$

In this way all components of $a$ are dimensionless and are required to be in the range zero to unity.

Introduce the least squares control function

$$
X^{2}(a)=N^{-1} \sum_{B=1}^{N}\left\{R^{-1} \sum_{i=1}^{R}\left(Y_{B}\left(x_{i}, a\right)-Y_{B}\left(x_{i}\right)\right)^{2}\right\},
$$

which provides a measure of average mismatch between observed and predicted bed positions. 
An iteration scheme which guarantees a closer correspondence of predicted and observed bed locations at each iteration, and which guarantees to keep each component of $a$ in the bounds $0 \leq a_{j} \leq 1$ can be constructed as follows: At the $n$th iteration represent the updated value $a_{j}(n+1)$ to $a_{j}$ by

$$
\begin{aligned}
a_{j}(n) & =\sin ^{2} \theta_{j}(n) \\
\theta_{j}(n+1) & =\theta_{j}(n) \exp \left[-\tanh \left\{\alpha_{j} \delta_{j}(n) \frac{\partial X^{2}(a(n))}{\partial a_{j}(n)}\right\}\right] \\
a_{j}(n+1) & =\sin ^{2} \theta_{j}(n+1)
\end{aligned}
$$

where

$$
\begin{gathered}
\alpha_{j}=\left|\frac{\partial X^{2}(a(0))}{\partial a_{j}(0)}\right|^{-1} \ln \left\{1+\left(M a_{j}(0)\right)^{-1}\right\} \\
\delta_{j}(n)=q_{j}(n) /\left[\frac{1}{P} \sum_{p=1}^{P} q_{p}(n)\right]
\end{gathered}
$$

with

$$
q_{j}(n)=\left(\left|a_{j}(n)-a_{j}(n-1)\right| / a_{j}(n)\right)+\beta^{2}
$$

where $M$ is the number of times the nonlinear iteration is to be undertaken, $a_{j}(0)$ is the initial choice for $a_{j}$ with $\left(0 \leq a_{j}(0) \leq 1\right), P$ is the number of parameters being varied, and $\beta$ is the scale-choice for calculating numerically the derivative $\partial X^{2} / \partial a_{j}$ (see below). The factor $\delta_{j}(n)$ chooses the most sensitive parameters for more rapid variation after the first iteration $(n \geq 2)$ while for $n=1, \delta_{j}(1)$ is set to unity. Note that if $a_{j}$ is chosen larger (smaller) than the value providing a minimum in $X^{2}$ then $\partial X^{2} / \partial a_{j}$ is positive (negative), in which case the exponential argument in Eq. (16a) is then negative (positive) so that the next updated value of $a_{j}$ is then decreased (increased). Hence the procedure always heads towards a minimum while keeping $a_{j}>0$.

Note also that the sensitivity factor $\delta_{j}$ will be small, $O\left(\beta^{2}\right)$, if $a_{j}(n) \approx a_{j}(n-1)$, and large, $O(1)$, if $a_{j}(n)$ is far from $a_{j}(n-1)$. Those components of $a$ that are not close to the values providing a minimum in $X^{2}$ are moved the most in a given iteration, while those close to providing a minimum in $X^{2}$ are hardly moved at all. Thus the procedure operates on the most sensitive parameters first.

Accordingly, the procedure increases (decreases) $a_{j}$ according as $\partial X^{2} / \partial a_{j}$ is negative (positive), guarantees to keep each component of $a$ in the range $0 \leq a_{j} \leq 1$, and to make the largest changes in those parameters that are furthest from satisfying the least squares control.

Two caveats exist: (i) the numerical accuracy with which a minimum in the least squares control function can be achieved is limited by the accuracy with which the partial derivatives, $\partial X^{2} / \partial a$, are calculated; (ii) the fact that the nonlinear iteration scheme guarantees to find $a$ minimum least squares mismatch is no guarantee that the procedure has found a global minimum.

The first caveat can be handled by decreasing the range of a particular component of the vector $a$ used to calculate a derivative: thus, with the approximation 


$$
\partial X^{2} / \partial a_{i}=\left(\beta a_{i}\right)^{-1}\left\{X^{2}\left(a_{1}, a_{2}, \ldots, a_{i}+\beta a_{i}, a_{i+1}, \ldots\right)-X^{2}\left(a_{1}, a_{2}, \ldots, a_{i}, a_{i+1}, \ldots\right)\right\}
$$

and a default value $\beta=0.1$, one can replace $\beta$ in turn by $10^{-2}, 10^{-3}, \ldots$, until some pre-set criterion is reached for accuracy of the derivative calculation. As a by-product, if numerical resolution allows $a_{i}(n)$ to exceed unity, then it is an appropriate strategy to immediately set $a_{i}(n)=0.9$, which forces $a_{i}(n)$ to be in the pre-set domain $\{0,1\}$, and which is also a valid value from which to calculate the partial derivative because, then, $a_{i}+0.1 a_{i}=0.99$, which is also in the required domain.

The second caveat is of greater concern because some procedure must be found for assessing whether multiple minima exist, the relative depths and parameter space ranges of such minima, and of deciding on which minimum is the appropriate value to use in parameter determination, from which one constructs the combined paleo-evolution of the fault and associated sedimentary beds. In addition, the number, $M$, of nonlinear iterations needs to be given.

A strategy, which has proven inordinately useful in other problems, where similar concerns have arisen, is as follows.

First, make a rough assessment of the minimum and maximum ranges that one anticipates will encompass each parameter. Quite often, there is enough geological knowledge to constrain approximately the ranges of initial estimates. When there is not enough such information, a broad linear search range for each parameter, done one order of magnitude at a time, is enough to provide quickly a fairly good initial estimate of parameter range. Then do a linear search over pairs of parameter values while holding the remaining parameters at their midpoint values. Two-dimensional plots of mean square control function contours in each two-parameter space then provide a rough idea of where minima lie, as well as specifying how to modify the initial estimated ranges of parameter values in order to improve the ability to focus resolution on the minima uncovered.

Then a multi-dimension nonlinear iterative search, as outlined above, improves the degree of fit between predicted and observed behaviors. The point here is that all parameters are allowed to vary simultaneously (rather than just two), thereby improving the overall parameter values. A linear search, as above, can be redone after the nonlinear iterations, so that one is more sure that a global minimum in the search ranges specified has been obtained. The pair of intertwined procedures (linear search, nonlinear iteration) can be repeated as many times as desired. The improvement in least squares mismatch using this type of strategy is considerable, as well as helping to ensure that the search procedure does not become "stuck" in a local minimum. The number, $M$, of nonlinear iterations to use is also fairly easy to determine. A group of 10 nonlinear iterations can be done, the "best" set of parameters determined, and then these parameters used as initial estimates for a further set of 10 nonlinear iterations. The ranges of the "best" parameters can be adjusted either by using an interleaved linear search, nonlinear iteration pair of procedures or, more automatically, by choosing those parameter ranges around the best value for which the mean square control function is a fixed percentage (10\% say) above the minimum value. In this way a sequence of a few lots of 10 nonlinear iterations at a time rapidly produces convergence to a minimum. 
The extent to which the Xiao-Suppe (1992) model of faulting and roll-over of structures is an appropriate representation of a given set of present-day observations can be addressed with the above nonlinear procedure.

The crucial point to note is that in the Xiao-Suppe (1992) type of model only three of the four angles are free to be specified, with the fourth being determined from these three as shown in Sec. II. On the other hand, there is no fundamental reason compelling an actually occurring fault to be precisely of the modeled form. A test for satisfaction of the proposed model fault behavior vis-à-vis a given set of observations of faulted sedimentary beds can then be made as follows.

First, assume that the Xiao-Suppe model is correct and so go through the inverse procedure given above to determine the best set of five parameters, and their uncertainties. Second, ignore the geometrical connection between the four angles required in the Xiao-Suppe model, and let all four of the angles, together with the SSR ratio and a fundamental length scale, $L$, be determined simultaneously and independently by an obvious generalization of the nonlinear inverse procedure given above, together with their ranges of uncertainty.

By direct insertion of the four angles so determined into Eq. (1), one can determine the extent to which Eq. (1) is, or is not, satisfied, thereby providing a direct measure of the ability of the Xiao-Suppe model to honor the particular set of observations. This procedure provides a method for testing the model.

The stability of a minimum to variations in data quality, quantity, and sampling frequency is also of concern. A minimum should be ruggedly stable, in the sense that small changes in the data values, number of datum points, and/or the distribution of the data should not seriously perturb either the existence of the minimum or the location of the minimum.

To address these concerns a small amount of random noise can be added to each datum point and the variation of the minimum addressed; in addition, different numbers of datum points can be added or removed in order to determine the number of datum points needed in order to provide a stable minimum. In this way an appreciation is obtained of the resolution and stability of the parameter values.

Taken together, the components of this general strategy are sufficient to permit a systematic and thorough investigation for self-consistent evolution of a fault and associated sedimentary beds within the framework of the proposed models of geometric fault pattern evolution.

Acknowledgments. The work reported here was supported by the Industrial Associates of the Basin Modeling Group at USC. Elizabeth Lorenzetti, Pete Brennan, and Marty Perlmutter, all of Texaco, are thanked for their critical and insightful comments on the work reported here.

\section{REFERENCES}

[1] E. A. Lorenzetti and P. A. Brennan, Graphical Tools for Interpreting Extensional Structures, Texaco E \& P Technology Department, Report 93-047, 48 pps., 1993 (available from the authors)

[2] H. Xiao and J. Suppe, Role of Compaction on the Listric Shape of Growth Normal Faults, AAPG Bulletin 73, 777-786 (1989)

[3] H. Xiao and J. Suppe, Origin of Rollover, AAPG Bulletin 76, 509-529 (1992) 